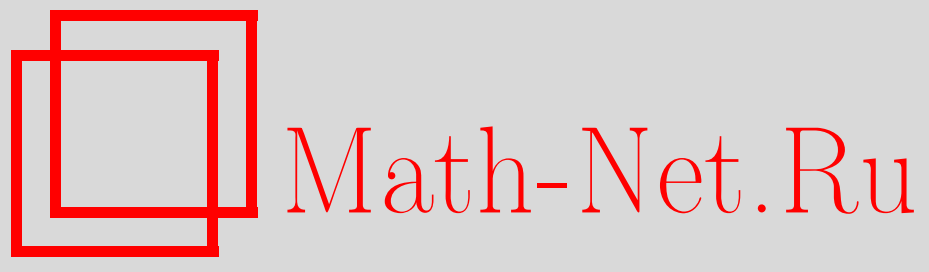

Г. Г. Ильюта, Результанты и деревья, УМН, 2007, том 62, выпуск 4, 161-162

DOI: https://doi.org/10.4213/rm7154

Использование Общероссийского математического портала Math-Net.Ru подразумевает, что вы прочитали и согласны с пользовательским соглашением http: //www . mathnet.ru/rus/agreement

Параметры загрузки:

IP: 54.209 .52 .79

26 апреля 2023 г., 13:20:14

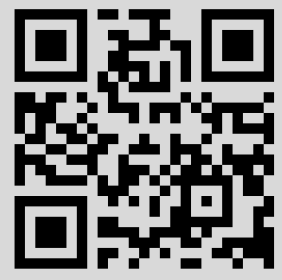




\section{Результанты и деревья}

\section{Г. Г. Ильюта}

Матричная теорема о деревьях (в классическом варианте представляющая число остовных деревьев графа как минор его матрицы Лапласа $L$ ) представляет минор матрицы с нулевой суммой столбцов как сумму весов деревьев в полном графе, веса ребер которого равны элементам матрицы (см. [1]). В детерминантных формулах для результанта многочленов $f_{0}, f_{1}, \ldots, f_{n}$ от $n$ переменных появляются матрицы вида $\left(\operatorname{res}_{\left(f_{1}, \ldots, f_{n}\right)}\left(g_{j} b_{i} f_{0}\right)\right)$ для некоторых многочленов $g_{j}$ и базиса $\left\{b_{i}\right\}$ в $C\left[x_{1}, \ldots, x_{n}\right] /\left(f_{1}, \ldots, f_{n}\right)[2]$. Добавляя к матрице строку и столбец, отвечающие любым многочленам $g_{0}$ и $b_{0}$, и домножая столбцы на коэффициенты их линейной зависимости, мы можем применить к результату матричную теорему о деревьях и представить результант как сумму весов деревьев. В заметке изучается наиболее близкий к теории графов частный случай такого подхода к вычислению результанта. А именно, мы обобщим в нескольких направлениях следующее замечание: матрица Лапласа полного $n$-графа с точностью до перестановки строк совпадает с матрицей Кронекера многочленов $c_{n-1}(x)=\left(x^{n}-1\right) /(x-1)$ и $c_{n-1}^{\prime}(x)$ (в частности, дискриминант многочлена $c_{n-1}(x)$ с точностью до знака равен $\left.n^{n-2}\right)$. Матрица Кронекера многочленов $f(x), g(x)(\operatorname{deg}(g)<\operatorname{deg}(f))$ - это матрица $\left(a_{i+j-2}\right)$, где $g / f=a_{0} x^{-1}+a_{1} x^{-2}+\cdots$; минор такой матрицы равен результанту [3]. Мы найдем графы, число деревьев в каждом из которых с точностью до знака равно результанту явно определенных многочленов. Для любого графа мы определим аналоги матриц Безу и Фробениуса [3], миноры которых равны числу деревьев графа. Матрице $L_{k}$, составленной из $k$-миноров матрицы Лапласа полного $n$-графа, мы придадим смысл в теории симплициальных комплексов (деревья обобщаются как ацикличные комплексы [4]) и в теории результантов. Задача: найти подобные интерпретации для результатов действия на матрицу Лапласа других симметризаторов Юнга.

Отметим еще несколько параллелей в теориях графов и результантов. Порядок критической группы графа (дискретного аналога якобиана римановой поверхности) равен числу его деревьев [5]. Аналогичная группа для результанта появляется в [6]. Аналог формулы $L=I I^{t}$ ( $I$ - матрица инцидентности графа) для дискриминанта имеется в [7]. Согласно [8], $\operatorname{Res}\left(f\left(x^{k}\right), g\left(x^{k}\right)\right)=(\operatorname{Res}(f(x), g(x)))^{k}$. Можно показать, что в теории графов эта формула отвечает следующему очевидному факту: сумма весов деревьев букета графов равна произведению сумм весов деревьев, составляющих букет графов. Матрица Кронекера для дискриминанта многочлена равна $V V^{t}$ ( $V$ - матрица Вандермонда для корней этого многочлена). Согласно [9], обобщенную матрицу $V$ для системы $x_{1}^{a_{1 i}} \cdots x_{n}^{a_{n i}}=1, i=1, \ldots, n$, можно интерпретировать как таблицу характеров абелевой группы. Из соотношений ортогональности для характеров вытекает, что $V V^{t}$ получается из диагональной матрицы перестановкой строк. Удаление корня $(1, \ldots, 1)$ приводит к вычитанию 1 из элементов матрицы $V V^{t}$, и мы приходим к матрице Лапласа полного графа.

Специализируя для $c_{n-1}(x)$ любую формулу для дискриминанта, получим формулу для числа деревьев полного $n$-графа. Интересно было бы найти комбинаторные доказательства этих формул, например, построить биекции между популярными в последние годы парковочными функциями и факторгруппами целочисленной решетки по действию матриц из детерминантных представлений дискриминанта. Сдвиг переменной на любое число $q$ не меняет результант, что позволяет включить формулы для $n^{n-2}$ в однопараметрические семейства (действие сдвига на матрицу Кронекера известно как $q$-биномиальное преобразование [10]).

Работа выполнена при поддержке грантов РФФИ 04-01-00762 и НШ-4719.2006.1. 
Для $S=\left(m_{n-1}, \ldots, m_{0}\right) \in\{0,1\}^{n}, 0 \leqslant k<n$, обозначим через $P_{k, S}$ многочлен $\left(x^{k} \sum m_{j}-\sum m_{j} x^{j}\right) /(x-1)$. Назовем $S k$-симметричным, если последовательность $m_{k-1}, \ldots, m_{0}, m_{n-1}, \ldots, m_{0}, m_{n-1}, \ldots, m_{k+1}$ центрально симметрична. Обозначим через $G_{k, S} n$-граф, в котором есть ребро $i j, i+j \leqslant n$, если и только если $(i+j-1)$-й элемент последовательности $m_{k-1}, \ldots, m_{0}, m_{n-1}, \ldots, m_{k+1}$ равен 1.

Предложение 1 . Если $S$-симметрично, то результант многочленов $c_{n-1}(x)$, $P_{k, S}(x)$ с точностъю до знака равен числу деревъев графа $G_{k, S}$.

Строки матрицы Петри $P$ графа [11] отвечают его $n$ нумерованным вершинам (ориентируем граф по убыванию номеров вершин), а столбцы - ребрам (парам вершин). В столбце $i j, i<j$, стоит 1 на местах $i, \ldots, j-1$ и 0 на оставшихся местах. Обозначим через $C_{n} n$-матрицу, у которой $\left(C_{n}\right)_{i i}=-\left(C_{n}\right)_{j j-1}=1$ для всех $i, j$ и остальные элементы равны 0. Очевидно, что $I=C_{n} P$. Назовем матрицей Безу (Фробениуса) графа матрицу $P P^{t}\left(P I^{t}\right)$ (матрицу Лапласа $I I^{t}$ в этом контексте можно назвать матрицей Кронекера графа). Названия объясняются формулами, связывающими матрицы Безу, Кронекера и Фробениуса пар многочленов $f, g$ и $f, 1$ [3], а также тем фактом, что матрица Кронекера (Безу) многочленов $c_{n}, 1$ с точностью до перестановки строк совпадает с $C_{n}\left(C_{n}{ }^{-1}\right)$.

ПредЛожениЕ 2. 1) Левые верхние ( $n-1)$-миноры матрии, Безу и Фробениуса графа равны числу деревъев этого графа. 2) Матрица Безу графа симметрична и ее элемент $і j$ для $i \leqslant j$ равен числу ребер ps, для которых $p \leqslant i \leqslant j<s .3)$ Элемент $i j$ матрицы Фробениуса граба равен разности числа ребер $i s, i<s$, для которых $j<s$, u числа ребер $p i, p<i$, для которых $p \leqslant j$.

Обозначим через $I_{k}$ матрицу инцидентности $(k-1)$-мерных и $k$-мерных граней в полном $k$-мерном комплексе на $n$ вершинах [4]. Аналогично работе [4], следующий факт можно обобщить и для деформированной матрицы Лапласа $L\left(q_{1}, \ldots, q_{n}\right)$ $\left(L\left(q_{1}, \ldots, q_{n}\right)_{i i}=\left(\sum q_{j}-q_{i}\right) q_{i}, L\left(q_{1}, \ldots, q_{n}\right)_{i j}=-q_{i} q_{j}, L(1, \ldots, 1)=L_{1}=L\right)$.

ПредЛОЖениЕ 3. $L_{k}=I_{k} I_{k}{ }^{t} \operatorname{diag}\left(n^{k-1}, \ldots, n^{k-1}\right)$.

Элементы матрицы, составленной из $k$-миноров матрицы Кронекера, можно определить аналогично элементам самой матрицы Кронекера, а именно, как коэффициенты некоторого ряда. Применяя тождество Бине-Коши к произведению матрицы $\left(a_{j-i}\right)$ размера $k \times \infty$ и матрицы $\left(x_{j}{ }^{-i}\right)$ размера $\infty \times k$, получим разложение функции $\left(g\left(x_{1}\right) \cdots g\left(x_{k}\right)\right) /\left(f\left(x_{1}\right) \cdots f\left(x_{k}\right)\right)$ в ряд по функциям Шура от $x_{1}{ }^{-1}, \ldots, x_{k}{ }^{-1}$ (частный случай ряда Ньютона [12]), среди коэффициентов которого находятся все $k$-миноры матрицы Кронекера.

\section{Список литературы}

[1] J. Moon, Discrete Math., 124:1-3 (1994), 163-171. [2] B. Mourrain, V. Pan, J. Complexity, 16:1 (2000), 110-180. [3] G. Orzech, Linear and Multilinear Algebra, 16:1-4 (1984), 275-282. [4] G. Kalai, Israel J. Math., 45:4 (1983), 337-351. [5] M. Baker, S. Norine, RiemannRoch and Abel-Jacobi on a finite graph, ArXiv: math.c0/0608360. [6] Д. К. Фаддеев, Записки науч. сем. ЛОМИ, 28 (1972), 164. [7] В. Parlett, Linear Algebra Appl., 355:1-3 (2002), 85-101. [8] J. McKay, S.-Sh. Wang, Arch. Math. (Basel), 53:4 (1989), 347-351. [9] P. Pedersen, B. Sturmfels, Algorithms in algebraic geometry and applications (Santander, Spain, 1994), Progr. Math., 143, Birkhäuser, Basel, 1996, 307-316. [10] M. Spivey, L. Steil, J. Integer Seq., 9:1 (2006), 06.1.1. [11] M. Gordon, E. Wilkinson, Pacific J. Math., 51:2 (1974), 451-453. [12] Г. Г. Ильюта, УMH, 60:2 (2005), 165-166.

Г. Г. Ильюта (G. G. Il'yuta)

Московский государственный открытый педагогический университет

E-mail: ilyuta@mccme.ru
Представлено С. М. Гусейн-Заде Принято редколлегией 08.05.2007 